\title{
LA INMEDIACIÓN DEL SUJETO COMO OBRA DE ARTE
}

\author{
Subject Propinquity as Art
}

\section{A imediação do sujeito como obra de arte}

\section{es}

\section{RESUMEN}

Pensar el ser humano desde una perspectiva estética y ética es acentuar ciertas operaciones que intervienen en la elaboración del yo. La construcción de una subjetividad se hace a partir de dos componentes que conforman la arquitectura estética: por un lado, ejercida como cuidado de sí, y por otro, la ética como el cuidado del otro. El despliegue de estos componentes tiene un lugar de expresión en la ciudad. En este sentido, el gobierno de la ciudad (que no es un lugar de poder institucional o político) se administra desde un sujeto que se elabora, que se talla a sí mismo como obra de arte. Este cotejo con el sí mismo es gobernabilidad que se hace en la cotidianidad del sujeto, en su vida íntima, a solas; cuando sale a la calle se puede encargar de la ciudad, cuidando del otro, ejerciendo gobernabilidad en la ciudad. Este sujeto es un ser moderno que se reinventa, que se crea ontologicamente, porque la dinámica más importante es el cuidado, el descubrimiento y la realización como obra de arte del sujeto.

PALABRAS CLAVE: estetica, ética, cuidado de sí, cuidado del otro.

\author{
Jalily Covezdy (Colombia) \\ Mágister en Estudios Políticos \\ Institución Universitaria Politécnico \\ Grancolombiano \\ jcovedzy@poligran.edu.co
}

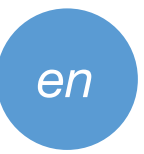

ABSTRACT

Picturing the human being from an esthetical and ethical perspective is to accent certain operations that intervene in the making of the self. The construction of subjectivity is made from two parts that form the aesthetic architecture: the care for the self and ethics as the care for the other. These components are expressed within the city (not a place with institutional or political power); and in this sense, its government is managed from a subject that sculpts itself as art. This comparison with the self is the daily nature governability carried out alone and in public while taking care of the other and by exercising governability in the city. This is a modern human being, reinvented, and ontologically created because the most important dynamic is the care, discovery, and realization as the subject's art.

KEYWORDS: Esthetic, ethic, self-care, care for the other.

\section{RESUMO}

Pensar o ser humano desde uma perspectiva estética e ética é acentuar certas operações que intervêm na elaboração do eu. A construção de uma subjetividade se faz a partir de dois componentes que conformam a arquitetura estética: por um lado, exercida como cuidado de si, e por outro, a ética como o cuidado do outro. O desdobramento destes componentes tem um lugar de expressão na cidade. Neste sentido, o governo da cidade (que não é um lugar de poder institucional ou político) administra-se desde um sujeito que se elabora, que se talha a si próprio como obra de arte. Este cotejo com o si próprio é governabilidade que se faz na cotidianidade do sujeito, na sua vida íntima, a sós; quando sai à rua pode-se encarregar da cidade, cuidando do outro, exercendo governabilidade na cidade. Este sujeito é um ser moderno que se reinventa, que se cria ontologicamente, porque a dinâmica mais importante é o cuidado, a descoberta e a realização como obra de arte do sujeito.

PALAVRAS CHAVE: : estética, ética, cuidado de si, cuidado do outro. 


\section{LA ÉSTETICA-ÉTICA COMO CONSTRUCCCIÓN DE CIUDADA- NÍA PARA COLOMBIA}

Jalily Covezdy

Cómo trasformar las microprácticas de poder vertical para formar ciudadanía? Develar esta inquietud requirió plantearse la condición de las relaciones entre los ciudadanos. La herencia cultural dejó la moral religiosa como el eje articulador, que ha posibilitado que se construya un orden vertical: la relación mando obediencia. En esa dinámica, el sujeto se encuentra escindido, y su puesta en el mundo es dicotómica (bueno-malo), su relación con él mismo es tormentosa y culposa. La relación con los otros es de orden-sumisón, una práctica de poder que relega a una condición de obediencia, una cultura de la humillación. Desde esta prespectiva, la ciudad es un espacio de encuentros conflictivos y la ciudanía una expresión de derechos y deberes que se imponen como obligación. Surge, después de varias disquicisiones, la perspectiva de lo estético que da cuenta de las relaciones. Una relación es el mundo de las subjetividades o de los sujetos que se actualizan en él. Estas están en una dinámica que se ha construido en Colombia y que tiene unos antecedentes implicatorios para que se den relaciones de orden vertical. Desmontar la verticalidad requiere una subjetividad que pueda incursionar en ella misma y definir un nuevo orden de cosas. Para lograrlo, se plantean la estética y la ética como caminos posibles desde las siguientes preguntas: ¿por qué una estética? ¿Ética y estética comparten el mismo lugar de análisis o reflexión?

Teniendo de base las anteriores preguntas, este ensayo se desliza hacia una elaboración y reflexión de lo estético-ético, político y humano. La perspectiva que se piensa respecto de estos conceptos se hace desde la cultura. La cultura que irrumpió y dio lugar a otras formas del ser humano, por lo menos el americano, y que se entiende desde las relaciones que lo establecieron y perviven en Colombia.

Panorama I

pp. 133-140।

Volumen 9 I

Número 17।

El fundamento teórico conceptual respecto de lo estético y lo ético se encuentra en autores, como Zygmunt Bauman, Felix Guattari y Michel Foucault, quienes, desde una posición teórico-filosófica, dan cuenta de ello.
Lo estético tiene que ver con el sujeto, es decir, con la construcción de la subjetividad y la elaboración de una identidad que se desarrolla desde el conocimiento que el individuo tiene acerca de sí mismo, el yo y su corporalidad. La estética ha sido un territorio de la filosofía y desde allí parte la pregunta por la belleza o lo bello.

Este concepto de belleza que podríamos pensar está referido a formas externas de lo bello y su relación con las obras de arte principalmente, se incorpora en esta mirada como planteamiento estético de las nuevas consideraciones del sujeto como obra de arte. Foucault, al referirse a esta forma de lo estético, lo desarrolla desde las

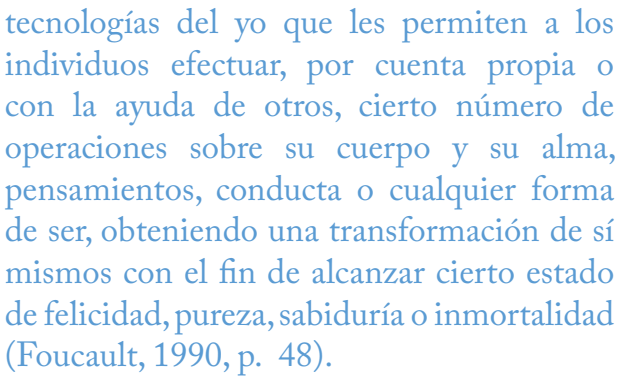

Vista así la estética es una elaboración en la que los sujetos son obras de arte. No está referida, en principio, a la postura, la decencia, las buenas maneras o las buenas costumbres. Tiene que ver, en cambio, con la transformación interior del sujeto, que se manifiesta en comportamientos, apreciación del mundo o formas de asumir el mundo desde la interioridad, la construcción de dignidad, la autonomía, entre otras.

Los griegos y romanos ya habían discurrido sobre este asunto, por lo menos Sócrates y su discípulo Platón.

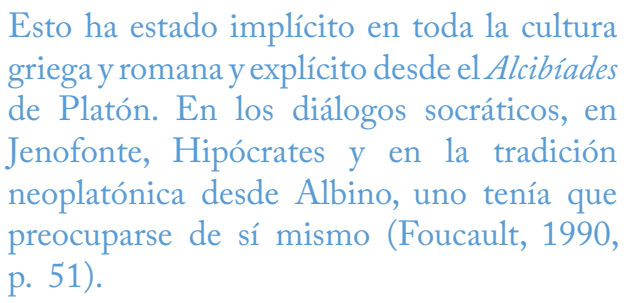

La preocupación por uno mismo o el sí mismo, epimeleia, es la forma de lo estético que abre dos elementos sobre los cuales se asienta la reflexión. El yo como principio de lo estético y la elaboración de la subjetividad:

Quisiera trazar el desarrollo de la hermenéutica del yo en dos contextos diferentes, históricamente contiguos: 1) la 
filosofía grecorromana en los dos primeros siglos a. C.) y 2) la espiritualidad cristiana y los principios monásticos desarrollados en el cuarto y quinto siglo al final del alto Imperio romano" (Foucault, 1990, p. 50).

El estudio sobre las tecnologías del yo arroja aproximaciones genealógicas muy importantes. A partir de los griegos, se busca el fundamento, el sustrato sobre el cual Occidente ha entendido o creído entender el yo. El yo no es una categoría del psicoanálisis freudiano, es más bien una mirada histórica, antropológica en la que el yo se elabora en el proceso de un encuentro consigo mismo, una preocupación por el desarrollo espiritual, cognitivo, corporal; hasta la nutrición tenía que ver con la forma como se entendía este yo.

Foucault lo precisa de la siguiente manera:

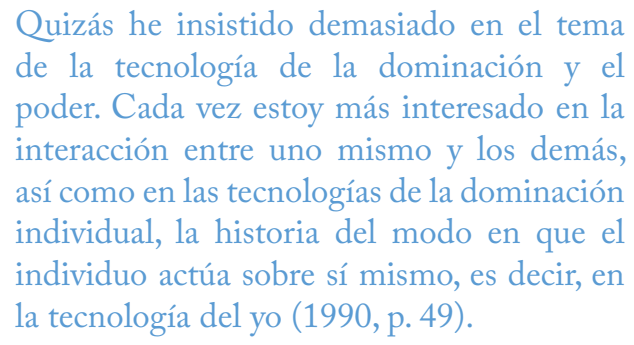

El arte o el estudio de saber quién soy pasa por las reflexiones sobre lo que el sujeto realiza con su cuerpo, su alma, pensamientos, conducta o cualquier forma de ser. El cuidado de sí, que es en el que estamos poniendo nuestro acento, cuando hablamos de lo estético, es una forma de introspección que invita al cuidado por uno mismo. Eso tiene que ver con los aspectos más importantes del ser humano: lo cognitivo, lo emocional, lo espiritual y lo corporal, por lo que a esos niveles se debe mover el cuidado de uno mismo. En este sentido, la inmediación del sujeto como obra de arte es lo que guía esta reflexión estética.

La tarea de pensarse incluye elementos muy importantes en el devenir creativo hacia una vida en que el cuidado de sí mismo tenga una importancia primordial como un ejercicio cognitivo o terapéutico, para alcanzar la excelencia humana, la sabiduría o el control sobre sí mismo; esta manera determinada de trabajar sobre la vida, semejante a la obra de arte, es la que va a dar lugar al concepto de gobernabilidad.

El gobierno de sí permite una perspectiva del desarrollo de una capacidad que en los griegos se ponía en juego en la ciudad. La gobernabilidad de sí es un aprendizaje para la vida política en la ciudad. La gobernabilidad de sí no es una experiencia mística de abandono ejercitándose para otra vida; por el contrario, esa lucha por la interioridad es para que en la calle, en la ciudad, se den nuevas prácticas de gobernabilidad.

El arte de la existencia, como lo despliega Foucault, es la construcción de una ética que sea una estética de la existencia. En ese sentido, la construcción de la estética encuentra dos campos importantes: el político como gobierno de sí mismo y la resistencia al poder que intenta gobernar al sujeto. Esta nueva subjetividad es la que le permite liberarse del Estado y de la individualización que va ligada a él; sobre esta afirmación se profundizará más adelante.

Foucault propone en su estética de la existencia un principio ya advertido por los griegos: el cuidado de uno mismo, ocuparse de uno mismo (heautou epemeleisthai). Tal es

El principio filosófico que predomina en
el mundo griego, helenístico y romano.
Sócrates encarna esta manera de filosofar
cuando interpela a la gente de la calle o a los
jóvenes del gimnasio y les dice: ¿Te ocupas
de ti mismo? (Foucault, 1990, p. 55).

Pero este ocuparse de sí mismo en primera instancia está ligado a un privilegio político. En ese sentido está unido al poder. La ocupación de sí mismo es porque hay debilidad hacia los placeres y los deseos, por lo cual debe ocuparse de sí mismo para gobernar la ciudad. Claro, cuando los placeres se vuelven ingobernables y se les otorga un principio de existencialidad. Esta es una relación importante, el individuo político es el que asume una ocupación de sí mismo en virtud de una expresión de su ocupación en la ciudad, en otro sentido asume el poder, o sea, su capacidad voluntaria como capacidad de poder.

Hay que entender que el placer no en sí mismo un problema para la ciudad, sino la forma o el lugar que ocupa el placer: el placer hay que regularlo. Si el placer o los placeres no se regulan, no hay desarrollo del sujeto, y
La inmediación del sujeto como obra de arte
I Panorama

I pp. 133-140

I Volumen 9

| Número 17 | Julio-diciembre | 2015 
menos política, porque la organización de los hombres es un asunto de la política.

En el Alcibiades, Foucault encuentra tres relaciones: 1) la relación entre la preocupación de sí y la vida política, 2) la relación entre preocuparse de sí y la educación y 3) la relación entre preocuparse de sí mismo y conocerse a sí mismo.

Estas podrían denominarse las preocupaciones de la modernidad o mejor las tareas de la modernidad, ya que la gubernamentalidad tiene que ver necesariamente con esta manera de ver la compleja relación sujeto, ciudad o país, si se quiere. Lo que ya se ha dicho, la relación estética que se expresa políticamente es entonces una manera de ser ético o ética. Y la preocupación de sí y la educación, porque es desde allí donde se elabora la esteticidad en una educación que se pronuncia en favor de la construcción de subjetividad, como una expresión del devenir estético del sujeto que se forma desde la pregunta ¿quién soy? Esta dinámica irrumpe en los pasillos de una escuela, que, por lo menos la colombiana, está quieta en el tiempo mientras que en los salones se producen las crisis, los desafíos, las lagunas, los destellos de un nuevo amanecer que aún es claroscuro.

La pregunta por el quién soy es la pregunta griega y moderna, contemporánea y sin tiempo. Es la pregunta por el conócete a ti mismo, y esto genera una profunda preocupación, porque de la filosofía que inicia la pregunta en la modernidad ha transitado a la psicología, poniendo la preocupación en el territorio de lo psíquico, sin desconocer sus importantes y profundos aportes.

La pregunta es interdisciplinar. En un mundo más complejo, la pregunta no es disciplinar sino multidisciplinar, compleja respecto de las relaciones que establecen en un conocimiento complejo. El conocimiento de sí es el

Panorama I

pp. 133-140 I

Volumen 9 ।

Número 17।

Julio-diciembre |

2015 |

\section{GENEALOGÍA DE UN CAMBIO}

En los siglos I y II o edad de oro, plantea Foucault, cambia la preocupación por sí mismo. El sí mismo se vuelve el objeto definitivo, lo cual quiere decir que ya no está abierto a la ciudad, a los otros, sino como encerrado en sí mismo. El cuidado de sí se dirige al alma pero desaloja al cuerpo, no se trata de prepararse para la política, sino, por el contrario, apartarse de ella.

De igual forma, en el cristianismo primitivo el cuidado de sí, en sentido socrático, está ausente, pues se debe renunciar a sí mismo para acceder a la salvación. En relación con Platón, los movimientos filosóficos del estoicismo conciben de manera diferente la verdad, la memoria y de examinarse a sí mismos. Hay para Foucault una relación de la verdad con el cuidado de sí mismo $y$, en ese sentido, también de la memoria como soporte de la bistoria de algo que no se vuelve a repetir, "desaparece el diálogo y aumenta la importancia de la cultura del silencio, del arte de la escucha" (Foucault, 1991b, p. 68).

Para los estoicos, la verdad no está en uno mismo, sino en los maestros, como ocurre con Platón, los logoi. Se memoriza lo escuchado para convertirlo en norma de conducta. En el estoicismo, el sí mismo se convierte en la preocupación por uno mismo o la actividad que uno ejerce sobre sí. Una estilización de la existencia. De esta manera, se amplía el concepto de lo estético a niveles más profundos, donde lo público y lo privado tiene lugar, que no se excluyen, sino que son necesarios para su existencia política.

Ha sido relevante mencionar estos dos acontecimientos históricos, porque en nuestro discurrir histórico ha quedado planteada una construcción del cuidado de sí más hacia una práctica no política que deja a la estética en el lugar de la obra de arte como objeto de contemplación, de la belleza perse. El sujeto construye su interioridad en una relación privada, y en su expresión pública él es otro sujeto. Una relación más fragmentada con el mundo, no armónica ni holística, herencia del cristianismo que tan determinante ha sido en nuestra construcción de mundo, de ser humano, de país.

\section{GUBERNAMENTALIDAD, ÉTICA Y MODERNIDAD ILUSTRADA}

Nos detenemos a mirar en este apartado cómo es que la gobernabilidad del sujeto o la estética del yo pasa a volverse gubernamentalidad en el discurso de la modernidad o la Ilustración, que es relevante en cuanto la aproximación es a revisar la categoría ciudadano o la práctica ciudadana que se espera tenga una articulación, un desarrollo desde la escuela. 
En el texto Seguridad, territorio, población, de Foucault (2011), aparece la constitución del concepto de gubernamentalidad. El autor hace mención de una economía específica del poder. Las sociedades han descentralizado el poder y los propios integrantes asumen el autogobierno. Pero en este caso hacia donde gira el planteamiento es hacia una sociedad del autogobierno.

En la sociedad, hay diferentes instituciones: la familia, la escuela, la prisión, entre otras, y cada una sigue una lógica de su propio gobierno, dinámica que permite un o algún conocimiento sobre los sujetos. Este concomimiento aprueba gobernar cómo los sujetos se comportarán desde su interioridad, desde el sujeto mismo. Lo que quiere decir que la gobernanza no es un asunto de afuera, de las élites, hegemonía, porque cada individuo reconoce su poder desde él mismo, desde una interioridad elaborada que se pone en juego en la sociedad, la familia, la escuela o las instituciones que refluyen sobre el propio sujeto. Las instituciones no son algo inamovible, fijo, quieto, están en cambio, y este es el aspecto más importante: los sujetos adecúan el entorno no ellos, sino a él. Aquí vemos cómo se desliza una nueva subjetividad.

La noción de gubernamentalidad es importante, porque permite poner el mérito de la libertad del sujeto y su relación con los otros, aquello que constituye la materia misma de la ética. Lo ético, por tanto, es un asunto que se da en relación con los otros, por lo que no es un asunto de la individualidad, como sí es lo estético. Tenemos y construimos eticidad, porque somos seres humanos, organismos vivos y dinámicos que regulan sus relaciones desde la interioridad de los sujetos.

La pregunta por lo ético es que esta conceptualización solo se da gracias a que existe el otro como receptor de esa experiencia inmediata, a que la responsabilidad de construir lo ético parte de una inquietud del sujeto por su propia condición de sí, su propia experiencia ética que incluye una reflexión sobre sí mismo. Lo cual tendrá impactos sobre los otros.

El cuidado de sí orienta gran parte del asunto de la existencia de la estética y, por tanto, de la resistencia como arte de existir. Y es importante la existencia de la estética porque interpreta lo planteado por Kant en el texto ¿¿Qué es la Ilustración? (2004) Y la describe como la forma moderna de la preocupación por nosotros mismos. Señala, en su interpretación, más una actitud que un periodo histórico, el presente, la actualidad, descubrir la naturaleza del presente y de "nosotros" en ese presente. Es exaltar el presente de forma práctica no contemplativa.

La modernidad, que es un concepto filosófico, sociológico e historiográfico, nos sirve para comprendernos, hay que sacarla de esas perspectivas y entenderla como actitud y como una profunda reflexión sobre el presente, sobre la actualidad, por lo que implica una ontología del sujeto que convoca esa realidad.

Hay que desprenderse de las condiciones que sujetan al individuo a una condición de dependencia frente a formas mitológicas de la vida y asumir la existencia, asumir el camino del poder, que es el camino de la creación humana. El ser humano es, ante todo, un creador, y desde allí es que se transforma en ser libre; esta es la forma de la existencia estética, la creación como una expresión de una interioridad elaborada que se expresa en sus formas de vida en él y en los otros.

La modernidad no es solo una forma de relación con el presente, es un modo de relación que el hombre debe establecer consigo mismo, es tomarse como objeto de una elaboración compleja y dura, ser moderno. E1 hombre moderno no parte solo del descubrimiento de sí mismo, sino que busca "inventarse a sí mismo". Elaborarnos a nosotros mismos y darnos nuestra propia actualidad es la tarea que nos impone la Ilustración.

La elaboración estética tiene una corresponsabilidad ética, ya que "no estamos solos en el universo", somos un cuerpo social y como tal la imperiosa necesidad, la interdependencia, nos convoca a una ética humana. Es en la modernidad donde el hombre ha logrado la gran conquista: ser libre. La libertad es, recordemos, una actitud y ser libre es, por tanto, la exigencia estética-política-humana.

Por tanto, la actitud moderna es un ascetismo que conlleva la tarea de hacer de nuestra vida una obra de arte. Debemos tener una atenta mirada a nuestro ser histórico, mirar las condiciones en las que se da y desarrolla la cultura europea-blanca, que impuso en América Latina un tipo de racionalidad política. Un ser político que se constituye desde el autoritarismo y paternalismo, que se articula en una sociedad clasista, desigual y sumamente 
Jalily Covezdy

Crítica permanente de nuestro ser histórico, es una de las aproximaciones de quien esculpe su propia obra de arte. Quiere decir esto que pensarnos y criticarnos es pensar la libertad y pensar nuestros límites. Este es un compromiso ético. No es la ética propuesta un decálogo de buenas costumbres.

En relacionarse con el otro hay también un compromiso: la denuncia o la revocatoria a las relaciones inequitativas, segregantes, excluyentes y marginales. Allí caben los excluidos y marginados, negros, pobres, discapacitados, indígenas, mujeres y todos aquellos que han sido negados desde una moralidad impuesta que no ha permitido la expresión de la libertad. La modernidad es más una actitud, un modo de relación en referencia a la actualidad; una escogencia voluntaria, una manera de sentir y de pensar, de actuar y de conducirse, que marca una pertenencia y se presenta como una tarea, que podemos denominar ética.

La modernidad como momento histórico, político, económico, cuestiona o problematiza la situación del presente y la constitución de sí mismo como sujeto autónomo, libre, sujeto de derechos que se piensa para comprender cómo lo que somos podría ser de otra manera; esta es la verdadera actitud ética. Esta nueva formulación estética-ética, la ontología de nosotros mismos en el presente, quiere decir una actitud crítica frente a nosotros mismos. Y frente a aquello que pensamos, sentimos y hacemos, tres condiciones del despliegue reflexivo-acción, lo cual quiere decir que debemos dejar de considerar la razón como totalidad.

Panorama I

pp. 133-140।

Volumen 9 ।

Número 17 I

Pensar, sentir y hacer son las formas como el ser humano ha de desplegarse creativamente, para no quedarse en el idealismo, en una vida ascética contemplativa, puesto que el hombre moderno y contemporáneo es un ser que se gobierna a sí mismo, que se define autónomamente, sin dependencias, resistente a todo aquello que el Estado promueve como individualidad, como estereotipo de la sociedad de consumo altamente domesticadora y enajenante del ser humano.
La relación con nosotros mismos es ontológicamente primera, lo cual significa una transformación de sí mismos en el pensamiento y en la vida. Preguntarse por la vida es una forma de actualizarse y ponerse en consideración de uno mismo. El cuidado de sí se presenta como exigencia y fuerza de creación de nosotros mismos y de nuestro mundo.

\section{RECHAZAR LO QUE SOMOS}

Debemos escapar de nosotros mismos para ser diferentes de lo que somos, pero esta diferencia debe estar entendida a la manera de Guattari (s. f.): rechazo de la individualidad que se nos ha impuesto durante siglos. Esta es Colombia, una cultura tejida en los avances de unos estereotipos culturales, que hay que descomponer en una nueva policromía cultural. No hay verdades absolutas, no hay verdad, por tanto, hay poliformas posibles de comprensión y realización.

Nos adormecimos en el tiempo mientras el mundo avanza, estamos fragmentados de ese mundo y de nosotros mismos, nuestras posibilidades históricas están en plena formación. La construcción de una nueva posibilidad está en el recurso más inmediato, la escuela y una crítica exhaustiva y un nuevo invento: una práctica de la creatividad debe aflorar, es nuestro aporte y propuesta.

Hay cosas que han venido cambiando lenta y caóticamente. Una sociedad en la que conviven diferentes momentos de la historia, zonas del país que viven en el siglo pasado. Ejemplo: hay aún regiones del país que no tienen luz, como algunos municipios de La Guajira; por otra parte, todavía existen prácticas campesinas en las que todavía se quema la tierra para poder empezar nuevas siembras. Son sitios que están a cincuenta años de atraso. Paradójicamente hay lugares en Colombia donde el avance es igual al de los países más desarrollados del planeta. Todas estas prácticas sociales dan cuenta e invitan al trabajo creativo y dinámico que hay que realizar como un país que está o navega en la modernidad. En la manera que se ha expuesto anteriormente la estética política humana puede potenciar esa actitud.

La ontología histórica de nosotros mismos nos propone como tarea de reflexión el análisis crítico del mundo en que vivimos. La primera estrategia es liberarnos de 
nosotros mismos, ya que la primera relación ontológica, ya se había dicho, es con nosotros.

\section{TOMAR CONCIENCIA DE NUESTRA PROPIA HISTORICIDAD}

Las preocupaciones por el cuidado de la vida en Colombia atraviesan por el dominio de una moral que ha sido desarrollada por la Iglesia católica y ahora por otras iglesias que abren posibilidades en la construcción de un ser humano plegado a las condiciones del decálogo y la moralidad judeo-cristina, del pastoreo, como diría Foucault.

Las preocupaciones por la salvación del alma y la exclusión del cuerpo. Cuando decimos conciencia de nuestra historicidad, es que la ética que hemos construido en Colombia pasa por la vigorización de la norma, el cumplimiento de ella de manera irreflexiva, sin que medie la estética y la ética en función del cuidado de sí mismo y del otro lo que ocasiona que no se resuelvan los problemas de la desigualdad que vivimos y tejemos entre clases sociales, estratos y otros modos de discriminación.

La construcción de la estética-ética es una formulación que debe conducir a criticar, profundamente, el origen de nuestra comodidad en todos los niveles sociales. Una comodidad que se esconde en la falta de asunción de lo político, una marginalidad en la mayoría de nosotros a esta responsabilidad como sujetos sociales. La estética-ética que proponemos está dirigida a la búsqueda de quién soy. Una pregunta profunda por el sí mismo en relación con cómo me pienso. Esto tiene que ver con la forma en que como ser humano ejerzo un gobierno de mí mismo, una gobernabilidad sobre la toma de decisiones, el proyecto de vida, la solución de problemas, la administración de la vida, el manejo del tiempo, el uso de los recursos, la forma como se establecen las relaciones, incluso la relación con la naturaleza. Esto es lo que significa pensar el ser humano. Una persona que tiene un estilo de vida que da cuenta de su propio quehacer en su vida.

Aquí proponemos una dimensión de lo ético: el lenguaje. La forma como hablamos, escuchamos y vemos, ya que estamos en una sociedad altamente visual, gracias a los medios masivos de comunicación y las tecnologías, como el celular, la fotografía y el video que cada vez tiene más importancia en nuestro ambiente social. No podemos ser seres pasivos frente a lo que escuchamos.
Las relaciones de poder vertical y asimétrico pueden transformarse en la medida en que nos hagamos conscientes de la forma como usamos nuestro recurso más importante: la palabra, en ese sentido, el lenguaje. Por eso, en la estética-ética, la palabra ocupa el lugar preeminente, porque nombrarnos iguales o desiguales es una formulación que se ha tejido desde un lado del discurso: el de los desiguales. Hay que impactar las nuevas relaciones de libertad con el poder de la igualdad política y existencial, estética/éticamente. Una transformación en los lenguajes de los sujetos.

El mal-trato, la comunicación despreciativa, la crítica, el chisme, la trampa, el ultraje, la violencia, la agresión, hacen parte de muchas de las formas como hablamos, como nos referimos a los otros, allí no establecemos un control del yo, entendido como práctica estética. Por ello, una de las principales dinámicas en la comunidad educativa debe ser cómo nos hablamos, establecer formas de hablar que enaltezcan la dignidad y potencien al sujeto.

Si revisáramos, en ese sentido, cómo han sido las formas de hablarnos en nuestra sociedad, el uso de lo difícil, del no puedo, de la víctima, de la carencia, de la infelicidad, entre otras, han sido palabras o frases dominantes, que impactan la dignidad, la desdibujan.

La dignidad ha de ser el ejercicio más importante en la construcción estética-ética, el valor que cada uno se dé a sí mismo. Es, por tanto, una valoración del poder que se posee, por lo que hay aquí un intento de comprender qué es este concepto tan potente y vitalizante. E1 valor existencial, que se refiere a la construcción de la autoestima, del autoconcepto, la identidad que se otorga cada uno depende del cuidado que se tenga de sí mismo. La violencia implícita y explícita que ronda en las familias, en las escuelas como recintos de poder vertical dominante y sancionatorio, relegan la dignidad o pliegan la dignidad al más fuerte, al que produce temor. Allí se dan también las reacciones, las resistencias, que Foucault establece como estéticas de la existencia. Las estéticas de la existencia son espacios donde los sujetos son creativos, propositivos, argumentativos, en el ejercicio de la libertad.

Estas estéticas son las estéticas del estar; aclaremos, estar es la única condición del ser humano, los demás seres vivos no saben de su existencia, el único que posee esa 
condición son los humanos. Ellos saben que viven, que coexisten, pero las condiciones socioeconómicas y políticas los han desvinculado de esta condición arrojándolos al individualismo, a la lucha por la supervivencia, en una sociedad fuertemente individualista y depredadora contradictoriamente de lo humano. Volvemos a traer la desigualdad como diciente de ese mal-estar social.

Estar presente, si se hacen los ejercicios ético-políticos, sujetos que están presentes en la vida social, porque están potenciados a partir de su elaboración, vidas en las que el arte no es una habilidad sino una acción sobre sí mismo, un accionar constante sobre el cuerpo, el pensamiento y las acciones que incluyen la relación con el otro y la naturaleza. Es decir, las relaciones son el fundamento de la arquitectura social, por lo que si ellas están en una balanza desequilibrante entonces lo que allí se articule está en ese orden; por tanto, el trabajo no es sobre los conceptos, es primero en el orden de las relaciones, y la principal relación es la del sujeto con él mismo, de allí siguen las demás.

Visto así, el cuidado de la dignidad es ejercicio político y resistencia estética, porque en el encuentro social, donde se dan las relaciones de poder, una persona que se empeña en su propia obra (existencia-vida) ejercita la dignidad cuando con otro actualiza su poder en la relación, lo cual quiere decir su capacidad (poder, dignidad, elaboración ético-estética).

Lo que está sucediendo es que de la dignidad transita hacia los derechos, que son los acuerdos que en la relación se establecen entre iguales, sucediendo un ejercicio de poder. Lo que en principio puede parecer una tensión, relación de fuerzas de poder, se despliega en la relación a una resistencia política de sujetos comprometidos en el respeto. Como diría Zuleta (1980), el respeto como aceptación de la diferencia, no tolerancia, aceptación del otro como diferente. Y sujetos dialógicos que hacen de la palabra la práctica más importante, porque se existe en la palabra, en lo que se dice, porque parte de sujetos que están en constante cuidado de sí, estética y éticamente.

La educación que se promueva en Colombia, en la familia y la escuela debe ser un compromiso ético-político, una función social de los ciudadanos. Los niños deben ser arrojados a una comprensión de su propia existencia

Volumen 9 ।

Número 17 |

Julio-diciembre |

2015 | a una elaboración de su vida (¿quiénes son?) antes que a saberes disciplinares, porque el principal acto educativo-pedagógico está en la relación del niño con su cuerpo, su pensamiento, sus emociones, sentimientos y su acción ontológica. Adultos que antes que exigir proponen desde una alteridad que se construyen en el ir y venir con el otro, con la subjetividad del otro.

Vista así, la subjetividad es una crítica, un rechazo al sí mismo constituido, a lo que no es lo más apto para la vida social, con los otros y la naturaleza. Una subjetividad que en los términos de hoy se resiste a las imposiciones del consumo y del egoísmo individual. Así dicho y fundamentado, es un desafío concebido como aventura, sueño y utopía.

\section{REFERENCIAS BIBLIOGRÁFICAS}

1. Foucault, M. (1990). Tecnologías del yo y otros textos afines. Barcelona: Paidós.

2. Foucault, M. (1991). Saber y verdad. Madrid : La Piqueta

3. Foucautl, M. (2011). Seguridad, territorio, población. Buenos Aires: Fondo de Cultura Económica.

4. Kant, I. (2004). ¿ Qué es la ilustración?: y otros escritos de ética, política y filosofía de la historia. Madrid: Alianza Editorial.

5. bligoo (s. f.). Guattari: el paradigma estético. Recuperado de http://patiotrasero.bligoo. com/content/view/215577/GUATTARIEL-PARADIGMA-ESTETICO.html\#. VsZKR8cfV7s

6. Zuleta, E. (2011). El elogio de la dificultad y otros ensayos (11. ${ }^{\mathrm{a}}$ ed.). Bogotá: Hombre Nuevo. 